\title{
Dementia in a community mental health unit in Spain: frequency by reason of referral
}

\begin{abstract}
Introduction: Despite the relevance of psychiatric symptoms in dementia patients, few studies are focused on dementia management by psychiatrist in community Mental Health Units (MHU) and $80 \%$ of Spanish psychiatrists recognize their lack of training on this field. The goals of this work are: 1) to analyze the frequency of dementia in a community MHU and 2) to analyze the risk of being diagnosed of dementia when geriatric patients are referred due to different psychiatric symptoms.
\end{abstract}

Method: Descriptive cross-sectional study of a sample of 211 "psycho geriatric" patients, selected between 2.222 patients referred to the MHU of the Hospital Clínico Universitario de Zaragoza in the course of 23 months. Inclusion criteria: patients older than 60 years with either: suspicion or diagnosis of dementia, first-onset behavior disorder or psychotic symptoms, and/or physical comorbidity.

Results: 84 patients were diagnosed of dementia $(39,8 \%$ of the selected sample and $3,8 \%$ of the overall sample of subjects referred to the MHU) $81 \%$ of Alzheimer type Dementia (AD). Patients referred due to Behavioral symptoms had a four-fold risk of being diagnosed of dementia, specifically $\mathrm{AD}$; and patients referred because of Psychotic symptoms had double risk of being diagnosed of dementia, risk was higher for other types of dementia (vs. AD).

Conclusion: According our results geriatric patients referred to a community MHU because behavioral and psychotic symptoms are frequently affected by dementia, so that it is necessary a better training on dementia diagnosis and management for psychiatrist. Studies describing the weight of dementia in Mental Health Services such this one will help psychiatrist to be aware of their important role on dementia management and health managers to better design strategies to improve healthcare for dementia which is considered one of the challenges of the century.

Keywords: community mental health unit, geriatric psychiatry, dementia, behavioral and psychological symptoms of dementia
Volume 2 Issue 4 - 2017

\author{
Patricia Gracia-García, 1,3 Marina Romance- \\ Aladren,' Beatriz Villagrasa,' Maria T Cortina- \\ Lacambra,' Miguel A Quintanilla-López, ${ }^{2,3}$ \\ Valero Pérez-Camo ${ }^{2,3}$ \\ 'Psychiatry Service, Hospital Clínico Universitario Miguel Servet, \\ Spain \\ 2Psychiatry Service, Hospital Clínico Universitario Lozano Blesa, \\ Spain \\ ${ }^{3}$ Universidad de Zaragoza, Spain
}

Correspondence: Patricia Gracia-García, Psychiatrist, Psychiatry Service in Hospital Universitario Miguel Servet, Zaragoza, Spain. Tel +34.876765 I08, Email pgraciag@salud.aragon.es

Received: July 25, 2016 | Published: November 01, 2017
Abbreviations: AD, alzheimer type dementia; BPSD, behavioral and psychological symptoms of dementia; CI, confidence interval; MCI, mild cognitive impairment; MHU, mental health unit; OR, odds-ratio; PG, psycho geriatric; S, syndrome; SD, standard deviation

\section{Introduction}

Despite behavioral and psychological symptoms of dementia (BPSD) appear in most demented patients over the course of the disease and that these symptoms have serious adverse consequences on patients and their caregivers ${ }^{1} 80 \%$ of Spanish psychiatrists recognize their lack of training and involvement on dementia care ${ }^{2}$ and previous reports describing dementia burden in general Mental Health Services are scarce. In this context the goals of this work are: 1) to analyze the frequency of dementia in a community Mental Health Unit (MHU) and 2) to analyze the risk of being diagnosed of dementia when geriatric patients are referred to the MHU due to different psychiatric symptoms.

\section{Material and methods}

Sample was recruited from patients referred to the MHU at the Hospital Clínico Universitario de Zaragoza, which covers attention for a population of 132.704 inhabitants, along a period of 23 months
(March 2015 to January 2017). 2.222 new patients older than 18 were referred to the MHU during this period, the most frequently (69\%) by their General Practitioner. A sample of 211 "psycho geriatric" (PG) patients was selected with the following inclusion criteria, defined by the team of the MHU: subjects older than 60 years + .

i. Psychiatric symptoms in patients with a diagnosed dementia

ii. Suspicion of cognitive impairment

iii. New-onset psychotic symptoms (including hallucinations, delusions, suspiciousness, confusional states) Behavioral disturbances (including restlessness, agitation/aggression, oposicionism, disinhibition)

iv. Depressive or anxiety symptoms in the context of a somatic illness

v. Complexity of management (substance use disorder, personality disorder, social problems...)

These patients were assessed by a same psychiatrist with formation and experience in geriatric patients.

Reasons for referral were assessed from the referral note and grouped according the most frequent psychiatric syndromes in geriatric patients. ${ }^{3}$ 

i. Affective Syndrome (S.): depression, euphoria, irritability
ii. Anxiety S: anxiety, worry, hypocondriasis, insomnia iii. Psychotic S: hallucinations, delusions, suspiciousness,
confusion
iv. Cognitive S: Memory or other cognitive troubles
v. Behavioral S.: restlessness, agitation/aggression, oppositionism, disinhibition

\section{vi. Other}

Diagnosis of cognitive impairment and dementia were made after psychiatric assessment (or delayed up to 6 months in doubtful cases) according to CIE-10 criteria. For the purposes of this work, we considered the following categories: 0) No demented, 1) Mild Cognitive Impairment (MCI) (F06.7 CIE-10); 2) Alzheimer type Dementia (AD) (F00 CIE-10); 3) other types of dementia (F01-03 CIE 10). Procedure of this study was approved by the Comité de Ética en Investigación de Aragón (CEICA) in February 2016 (CP-CI PI 16/0031). For statistical analysis we used the SPSS program. A descriptive, transversal analysis of data was made through frequency tables and logistic regression analysis, calculating (respectively) the statistics chi-square and odds-ratio (OR).

\section{Results}

PG patients included in the study $(\mathrm{n}=211)$ had a mean age of 77,9 (Standard Deviation (SD) 8,1) and 62,1\% ( $\mathrm{n}=133$ ) were women. Reasons for referral were distributed as follows: $29,9 \%$ Behavioral S., 26,5\% Affective S., 20,4\% Psychotic S., 11,4\% Anxiety S., 6,2\% Cognitive S., and $5,4 \%$ other reasons.

After psychiatric assessment, 84 patients were diagnosed of dementia $(39,8 \%$ of the selected, $\mathrm{PG}$, sample and $3,8 \%$ of the overall sample of subjects referred to the MHU), 68 of them $(80,9 \%)$ accomplished criteria for AD. Other 19 patients $(8,9 \%)$ were diagnosed of MCI. Patients with dementia diagnosis were significantly older (mean age 80,4; SD 8,1) than non-demented patients (mean age 76,3; SD 7,4) $(p=0,000)$, we did not find differences in gender by diagnostic group.

The figure shows frequency of diagnosis of dementia by reason of referral, comparing with diagnosis of dementia in the rest of the PG sample (Figure 1).

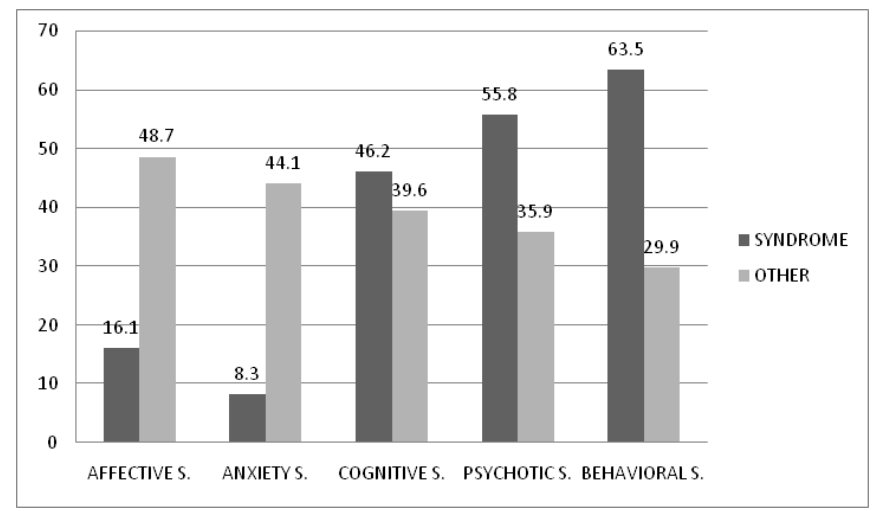

Figure I Frequency (\%) of dementia diagnosis in patients referred by symptoms included in each Syndrome (S.), related to all other patients in the "psycho geriatric" (PG) sample.
Patients referred due to Affective S. and Anxiety S. had lower risk of being diagnosed of dementia comparing with patients referred by other reasons (Odds-Ratio (OR) 0,2 (Confidence Interval (CI) 95\% $0,1-0,4)$; and OR 0,1 (CI95\% 0,0-0,5), respectively). Patients referred due to Behavior S. and Psychotic S. had significantly higher risk of being diagnosed of dementia ( $\mathrm{OR}=2,2$ (CI95\% 1,1-4,4); and OR 4,1 (CI95\% 2,2-7,6), respectively). Patients referred due to Cognitive S. did not have higher risk of being diagnosed of dementia, but they have higher risk of being diagnosed of MCI (OR 8,2 (CI95\% 2,4-28,3). After controlling by age, results remained significant.

Regarding differential risk by type of dementia, we found that patients referred to the MHU because Psychotic S. had double risk of being diagnosed of $\mathrm{AD}(\mathrm{CI} 95 \%$ 1,0-4,2) and a risk 3,4 times higher of being diagnosed of other type of dementia (CI95\% 1,1-10,4). Patients referred because Behavioral S. had a 5 times higher risk of being diagnosed of $\mathrm{AD}(\mathrm{CI} 95 \%$ 2,6-9,7) and a 1,5 higher risk of being diagnosed of other type of dementia, but in this case results were not statistically significant (CI95\% 0,4-5,0).

\section{Discussion}

According our results, we estimate that $3,8 \%$ of adult population referred to our MHU suffers from dementia. In our PG sample frequency of dementia is $39,8 \%$, similar than the frequency previously reported in community psycho geriatric settings in other countries $(35,7 \%){ }^{4}$ Previous studies reported that depression ${ }^{2}$ and "negative type" symptoms (apathy, anergia, restriction in activity and anhedonia ${ }^{5}$ are the SPCD most frequent in demented patients, especially in prodromal and mild stages of the illness. ${ }^{6}$ Despite we cannot infer from our data an association between symptoms justifying referral and stage of the illness, PG patients referred to the MHU by depression had lower risk of being diagnosed of dementia than patients referred by other reasons. On the other side, $\mathrm{PG}$ patients referred by behavioral and psychotic symptoms had higher risk of being diagnosed of dementia. We could hypothesize that demented patients are referred to the MHU in a more advanced stage of illness, but also that behavioral and psychotic symptoms are more disruptive for caregivers; so that they justify referral of demented patients to MHU more frequently than affective symptoms. ${ }^{7}$ The last hypothesis would be consistent with the opinion of Spanish psychiatrist, who consider that they only attend the most complex dementia patients. ${ }^{1}$

A possible concern regarding our study might be generalizability of our results, due to the fact that it is focused in only one MHU. But notice that our MHU is reference for a large population from the city of Zaragoza (Spain) and surroundings and that the period of inclusion of patients is quite large, so we consider that our results could be representative and comparable with other MHU in our country. Our study has other limitations

i. We cannot infer prevalence data of dementia, only frequency of dementia in subjects referred to the MHU because suffering from psychiatric symptoms.

ii. We only analyzed in our study psychiatric symptoms that have justified referral to the MHU but patients could suffer from other symptoms not systematically assessed for research.

iii. We only selected in our PG sample patients older than 60 years presenting some inclusion criteria, established by our MHU team, according to information registered at the referral note. 
This selection bias should be taking in account when interpreting our results: on the one side, frequency of dementia in our MHU might have been underestimated and, on the other side, our inclusion criteria prioritize patients referred to the MHU because cognitive, behavioral and psychotic symptoms over patients referred because depressive or anxiety symptoms. However, results of our work emphasize that geriatric patients referred to the MHU because behavioral and psychotic symptoms should be assessed by psychiatrist with specific training on dementia. Future descriptive studies with less restrictive criteria of selection and using standardized instruments for assessment of psychiatric symptoms should be suitable.

\section{Conclusion}

According our results, geriatric patients referred to a community MHU because behavioral and psychotic symptoms are frequently affected by dementia so that it is necessary a better specific training on dementia diagnosis and management for psychiatrist. In some European, developed countries such as Spain, there is an underdevelopment of specific psychiatric resources for geriatric patients and psychiatrist involvement in the management of dementias is limited ${ }^{1}$ despite it is recognized their necessary involvement in multidisciplinary teams for global care of demented patients by Clinical Guidelines. ${ }^{8}$ Studies describing the weight of dementia in Mental Health Services such this one will help psychiatrist to be aware of their important role on dementia management and health managers to better design strategies to improve healthcare for dementia, which is considered one of the challenges of the century.

\section{Acknowledgements}

None.

\section{Conflict of interest}

Authors declare that there are no conflicts of interest.

\section{References}

1. Lyketsos CG, Lopez O, Jones B, et al. Prevalence of Neuropsychiatric Symptoms in Dementia and Mild Cognitive Impairment. JAMA. 2002;288(12):1475-1483.

2. Martín CM, Arranz FJ. Perspectiva De Los Psiquiatras Españoles Respecto a La Atención De Las Demencias. La Encuesta Psico Dem. Rev Psiquiatr Salud Ment. 2015;8(1):17-25.

3. Sánchez PM. In: Sociedad Española de Psicogeriatría. Guía Esencial de psicogeriatría, 2nd ed. panamericana.2014.

4. Holroyd S, Duryee JJ. Characteristics of persons utilizing a geriatric psychiatry outpatient clinic. J Geriatr Psychiatry Neurol. 1997;10(4):136-141

5. Saz P, López-Antón R, Dewey ME, et al. Prevalence and implications of psychopathological non-cognitive symptoms in dementia. Acta Psychiatr Scand. 2009;119(2):107-116.

6. Stella F. Neuropsychiatric Symptoms in Alzheimer's disease Patients: Improving the Diagnosis. J Alzheimers Dis Parkinsonism. 2014;4:146.

7. Martín CM. Síntomas Psiquiátricos De Las Demencias. Informaciones Psiquiátricas Tercer Trimestre. 2007;189:1-14.

8. Grupo de trabajo de la Guía de Práctica Clínica (GPC). sobre la Atención Integral a las Personas con Enfermedad de Alzheimer y otras Demencias. 2010 . 